\title{
Personalized 3D Breast Cancer Models with Automatic Image Segmentation and Registration
}

\author{
Sílvia BESSA*1,2, João TEIXEIRA ${ }^{1,2}$, Pedro H. CARVALHO ${ }^{1}$, \\ Pedro F. GOUVEIA ${ }^{3,4}$, Hélder P. OLIVEIRA ${ }^{1,2}$ \\ 1 INESC TEC, Porto, Portugal; \\ 2 University of Porto, Porto, Portugal; \\ ${ }^{3}$ Champalimaud Foundation, Lisbon, Portugal \\ ${ }^{4}$ Medical School, University of Lisbon, Lisbon, Portugal
}

https://doi.org/10.15221/20.15

\begin{abstract}
Breast conservative surgery (BCS) combined with radiotherapy has become the treatment of choice for the majority of women suffering from breast cancer. Every year approximately 2.1 million new cases arise worldwide, but fortunately the 10-year survival rate now exceeds $80 \%$ mostly due to the early detection of non-palpable breast cancer. The accurate localization of the tumor is of utmost importance to decrease the re-excision rate and necessity of a second surgery, and also pivotal to the minimization of unaesthetic outcomes caused by these interventions. Nevertheless, 7 out of 10 patients will need invasive pre-operative localization and women will live long lives with the dire consequences of cancer treatments. Currently, it is up to the physicians to correlate multimodal 2-dimensial (2D) sectional images to the 3D space for surgical planning. The challenge is now focused on the technology to create an alternative non-invasive tumor location procedure that can be ethical and fit to the clinical set. This paper describes an automatic image segmentation and registration algorithm that fuses 3D optical scans of the breast surface, with interior radiological data for tumor characterization, to build a personalized digital breast model that can potentially be used as a non-invasive digital pre-operative localization technique, improving tumor visualization and surgery planning.
\end{abstract}

Keywords: breast cancer, 3D breast model, fusion, multimodal registration, automatic segmentation, magnetic resonance image, surface, tumor location

\section{Introduction}

Conservative surgery is the most common treatment for women with non-palpable breast cancer or relatively small lesions. The main challenge of this surgery is to obtain free margins while minimizing the resection of healthy tissue to potentiate good aesthetic results. Therefore, a requirement for successful breast conservative surgery (BCS) is a careful preoperative planning with the location and spread of tumor accurately determined, especially in non-palpable lesions [1]. The location and spread of breast cancer are characterized using radiological medical imagining - mammography, ultrasound (US) and MRI - but surgery planning considers both interior and surface information of the breast to better plan the removal of lesions and discuss the aesthetic outcomes of different surgery options with the patient.

Methods for preoperative localization of breast lesions have evolved over time: wire guided surgery is the most common method. Other localization techniques can also be used, such as radioactive seeds, carbon tattooing or ultrasound guided clips, but they are all invasive procedures [2]. Recent innovations in 3D surface imaging technology at affordable prices have paved the way to create personalized breast cancer models as an alternative non-invasive tumor localization procedure. These models are digital representations that provide accurate tumor locations on 3D optical scans of the breast surface, already accounting for the different breast shape deformations caused by the multi-modal radiological and surface imaging modalities, acquired in different instants of time and settings.

The creation of these models depends heavily on the advances and performance of multi-modal breast image registration algorithms, which have primordially been focused on the correct mapping of lesions and radiological anatomical structures, without integrating 3D surface scans [3]. 
In [4], we proposed a multimodal fusion algorithm that combines prone MRI with 3D surface scans in upright, that was thoroughly validated [5] in its ability to create adequate personalized 3D models of the patient's breast with precise tumor location. However, this strategy relied on manual annotations of the breast anterior contour on the T1-weighted MRI (MRI-T1w) sequence, where the 3D anatomical landmarks and structures are most visible. This lengthy and tedious task enabled a fair assessment of the performance of the fusion algorithm independent of segmentation errors, but it is not compatible with the clinical set. Moreover, tumors were actually annotated on the Dynamic Contrast-Enhanced MRI (DCE-MRI, Dyn). DCE-MRI provides an accurate delineation of the shape and extent of the tumor and offers the highest sensitivity for ductal carcinoma in situ (DCIS), which spreads in three-dimensional directions [6]. Consequently, this urged the necessity of fusing volumetric data of T1w and Dyn sequences to obtain an interior model of the breast [7]. The rigid registration of those radiological volumes relied on automatic segmentation of the breast contour on both sequences.

The natural extension of these works is the development of a fully automatic MRI/3D scan fusion algorithm using automatic segmentations, which we describe here.

\section{Patients and Methods}

Twenty-four patients with a Tis/T1-T3 breast cancer proposed for breast conservative treatment at the Champalimaud Clinical Centre, Portugal, between April 2017 and January 2019 were selected and included in the current study. Contraindications included T4 cancers, inflammatory carcinoma and breast recurrence post-breast conservative treatment. After written informed consent, all patients were proposed for image acquisition and anatomical measurements (Fig. 1):

- Surface data: 3D optical scans of the patient torso in the standing position with hands on hips, capturing the size and shape of the breasts using Go!Scan 3D by Creaform ${ }^{\circledR, 1}$

- MRI (Philips Ingenia 3T) with gadolinium contrast was performed according to institutional protocols - patient in prone position with arms up - acquisition of T1w and Dyn sequences:

- MRI-T1w: 60 slices $(3 \mathrm{~mm}), 720 \times 720$ pixels $(0.3-0.5 \mathrm{~mm} / \mathrm{px})$

- DCE-MRI: 300 slices $(1 \mathrm{~mm}), 300 \times 300$ pixels $(0.5-0.6 \mathrm{~mm} / \mathrm{px})$

- Annotation of the MRI tissue portions (with T1 and sd3 image sequences) were performed and validated by two radiologists using the Horos ${ }^{\circledR}$ software v2.4.0. for a subset of 10 patients. These patients integrated the dataset used in [5] to evaluate the benefits of incorporating a biomechanical model (BM) of pose transformation on the fusion algorithm pipeline. In detail, for this study both manual annotations of breast contour from MRI-T1w and breast tissue including malignant tumor(s) from DCE-MRI were used for validation.
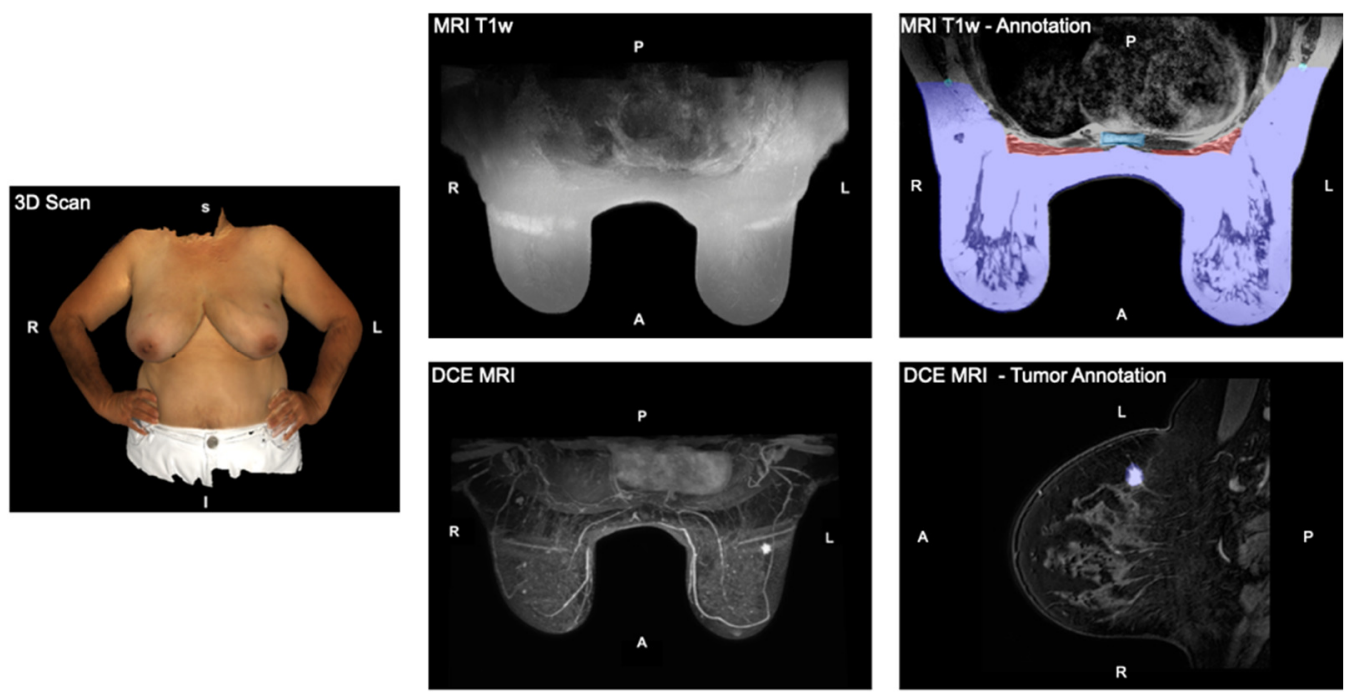

Fig. 1 - Image acquisition and anatomical annotations: 3D surface scan in upright (left); Maximum intensity projection of MRI-T1w acquired in prone position, scanning in the axial plane (center top) and DCE-MRI in prone position, scanning in the sagittal plane (center bottom); 2D sectional images of MRI-T1W and DCE-MRI with manual annotations. Legend: P- Posterior, A - Anterior, $R$ - Right, L - Left, S - Superior, I - Inferior.

\footnotetext{
${ }^{1}$ https://www.creaform3d.com/en/handheld-portable-3d-scanner-goscan-3d
} 


\subsection{Automatic Segmentation of Breast Contour}

Breast MRI images are sequences of 2D sectional images that depict the main breast anatomical structures and emphasize lesions. Following clinical protocols, breast MRI images are acquired with the patient in prone position with the arms up, with coils pressing the breast tissue. MRI-T1 sequences facilitate the identification of anatomical landmarks such as sternum, pectoral and latissimus dorsi muscles and clavicules, while 3D tumor morphology and spread are mostly assessed on DCE-MRI, which takes advantage of the injection of contrast to evidentiate the tumor shape, size, heterogeneity and margins [6].

Although the patient positioning is the same for both sequences, cross sectional images are acquired in different directions: MRI-T1w has an axial acquisition, while and DCE-MRI slices are on the sagittal plane. Any sequence produces a radiological volume that can be observed from any anatomical plane perspective, but this difference in acquisition has implications on both the field of view (Fig.1, 2D sectional images) and resolution. Therefore, the fusing of their information results in a 3D radiological volume, with higher resolution and larger field of view. Tumors are well categorized and the radiological volume includes rigid/quasi-rigid anatomical landmarks, which are shared between interior and surface data. This facilitates registration tasks with other modalities (radiological or 3D optical scans).

In [7], breast anterior surfaces were automatically segmented on both MRI-T1w and DCE-MRI sequences, using the same segmentation pipeline: a Canny edge detector applied to both coronal and sagittal perspectives to obtain a 3D coronal mask, followed by morphological operations and a ChanVese level-set block to refine the breast contour of each slice to obtain the 3D breast surface. The resulting breast surfaces were then rigidly registered using a regularized Iterative Closest Point (ICP) algorithm to complement anatomical reference points obtained from MRI-T, with lesion annotations from DCE-MRI. This framework for fusion of T1w and DCE-MRI sequences was designed to transpose precise tumor annotations from DCE to the MRI-T1 volume. Consequently, breast surface segmentations were optimized to minimize the registration errors by imposing a strict stop criteria: stopping after both left and right breast surfaces merged or the area between the segmentations of sequential coronal slices stopped increasing.

Fig. 2 provides a comparison between the breast anterior surface annotation on MRI-T1w exported as a white point cloud (PCL), with the automatic segmentation PCL from the same sequence (orange) used to drive the radiological matching of T1w and DCE sequences. Noticeably, the original automatic segmentation from [7] contains less information than the manual annotation, hencefore referred to as GT.

However, the MRI/3D fusion algorithm from [5] was designed to take advantage of a GT annotation that includes a wider portion of the torso on the coronal plane, including quasi-rigid anatomic strucutres that facilitate the matching of breast surfaces from MRI (Fig.2, GT) and 3D surface scans (Fig.1, 3D Scan). This motivated an adaptation of the stop criteria imposed on the segmentation algorithm and the addition of an extra post-processing step of the 3D volumetric mask to remove undesired internal structures segmented with the breast contour.

The schematic of the new segmentation pipeline is shown in Fig.3, with the new blocks highlighted in orange. In detail, the three top and bottom slices of the MRI-T1w sequences were used to estimate the parameters of an ellipse that fits the patient's torso in each slice. The centroids of the resulting ellipses were averaged and the coronal coordinate of the average centroid was used as the new stop criteria. This lead to the inclusion of partial segmentations of other anatomical structures, which demanded cleaning the the interior of the torso and the reconnection of the breast surface. This extra postprocessing step was applied to the coronal mask, and comprised an enhancement of breast contour (morphological dilation and thining added to the original segmentation mask), followed by the definition of lateral boundaries of the breast contour (left and right limits) and a 3D connected components on the volumetric mask (kernal size $=1$ pixel). The new segmentation of the MRI-T1w breast surface is closer to the GT, as shown on Fig.2 (new segmentaiton, blue PCL). 


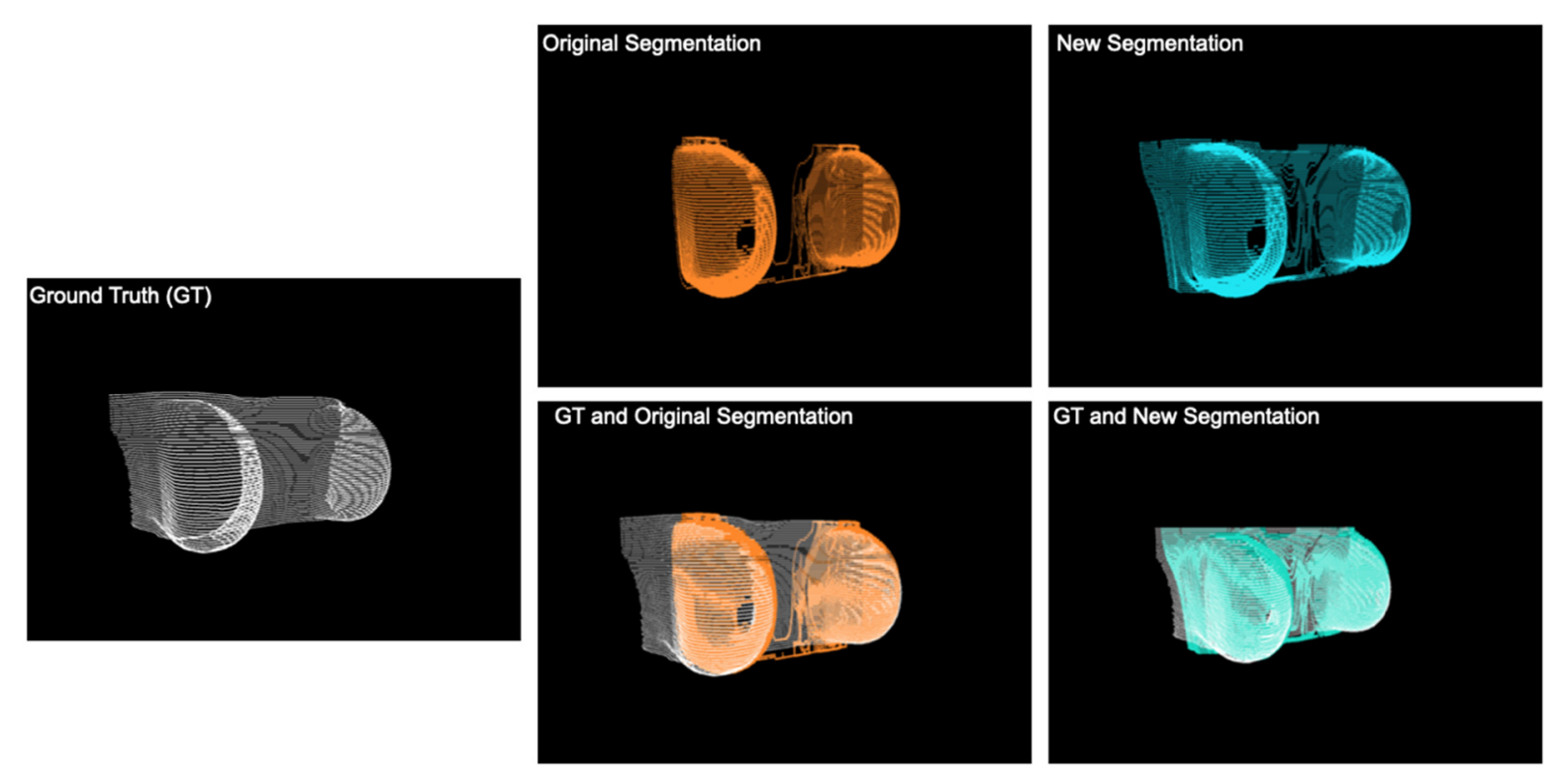

Fig. 2 - Comparison of the automatic segmentations of breast anterior surfaces (orange and blue) with the manual annotation (white).

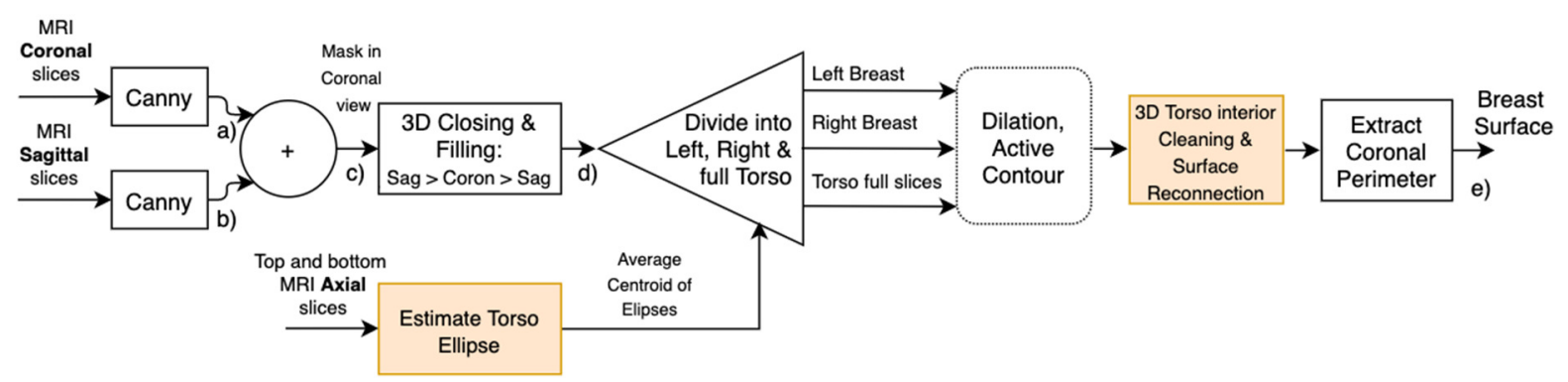

Fig. 3 - Automatic Segmentation Algorithm: orange boxes show the new modules added to obtain breast surface segmentations closers to the GT.

\subsection{Multimodal Registration and Validation}

Literature on breast image registration is quite extensive and mostly tailored to merge radiological exams, with notable contributions to computer assisted diagnosis, visualization and surgery planning. The next logical step was then the inclusion of newly available data, such as 3D surface scan. The underlying idea being the creation of individualized 3D breast cancer models that allow the visualization of the tumor inside of the digital breast. Literature on this topic is sparser [3], and mostly resorts to BMs of pose transformation - computationally complex physical models often generalized from datasets of few patients - to recover large breast deformations caused by variations of the patient position. Moreover, those works usually map prone MRI (or a supine variation) to 3D surface scans of the patient on the surgical position (supine).

However, surgery planning transcends the clinical team, with increasing integration of the patient on the decision process. In fact, proper communication of expected aesthetic outcomes of different treatment options plays a major role on the acceptance of the long-term consequences of those treatments [8]. Moreover, patients have difficulties projecting themselves on 3D models that are not in the position they see themselves in the mirror (upright position). The prediction of shape deformations of the breast following BCS has also attracted the interest of the artificial intelligence and computer vision communities which designed BMs [9] or trained machine learning algorithms [10] to show expected surgery outcomes on upright personalized 3D models of breast that the patient can relate to.

In [4,5], a multi-modal image registration combines MRI/3D surface scans into a patient-specific breast model that enables precise non-invasive pre-operative location of the tumor and provides a proper 3D upright representation for computer assisted surgery planning [10]. This two-step strategy comprises ICP for initial alignment of interior and surface data, and free form deformation (FFD) for the fine 
matching of the breast anterior surfaces in both representations. It was thoroughly validated with a new image acquisition protocol that included breast surface markers in both, MRI and 3D scans, to compute target registration errors (TRE, gold standard). The tumor location was also validated in comparison to the widely used carbon tattoo marking and clinical reports. The single-breast MRI/3D fusion algorithm increased tumor location accuracy compared to a biomechanical simulation and was not affected by variances in breast volume. Best TRE $(18.5 \pm 3.88 \mathrm{~mm})$ were observed with the inclusion of the BM of pose transformation, but tumor locations were consistently worse ( $80 \%$ of the cases) than fusion results without BM (TRE of $26.26 \pm 6.61 \mathrm{~mm}$ ).

The reported results were obtained with manual annotations of the breast surface on MRI-T1w, with the objective to discard the influence of segmentation errors in the fusion algorithm validation. Now, the same fusion algorithm is applied to an extended subset of patients, using fully automatic segmentations of breast surface resultant from the adapted segmentation pipeline described in the previous section.

Fig.4 shows the results of each fusion algorithm step using these new automatic segmentations to create personalized 3D breast models in a fast and automatic way.

Once more, the accuracy of pre-operative tumor location provided by these non-invasive 3D breast cancer models was validated with our clinical partners, including the surgeon that treated all the patients included on this study. For each patient, the surgeon visualized and manipulated the patient-specific 3D model using the Meshlab software, and resorted to all available radiological images, clinical reports, carbon tattooing marks (when available) and personal notes of the surgery to answer the following questions:

1. Correct Quadrant: is the tumor located on the correct quadrant (4 quadrants plus 4 quadrant intersections)?

2. Overlap: Does the tumor overlap the carbon mark?

3. Acceptable Location: Is the tumor location acceptable/ could the surgeon perform the same incision used in surgery to resect the tumor?

Fig. 5 shows a 3D breast cancer model of patient alongside with a schematic of the surgery annotation, as an example of the subset of information available to the surgeon during the validation task.
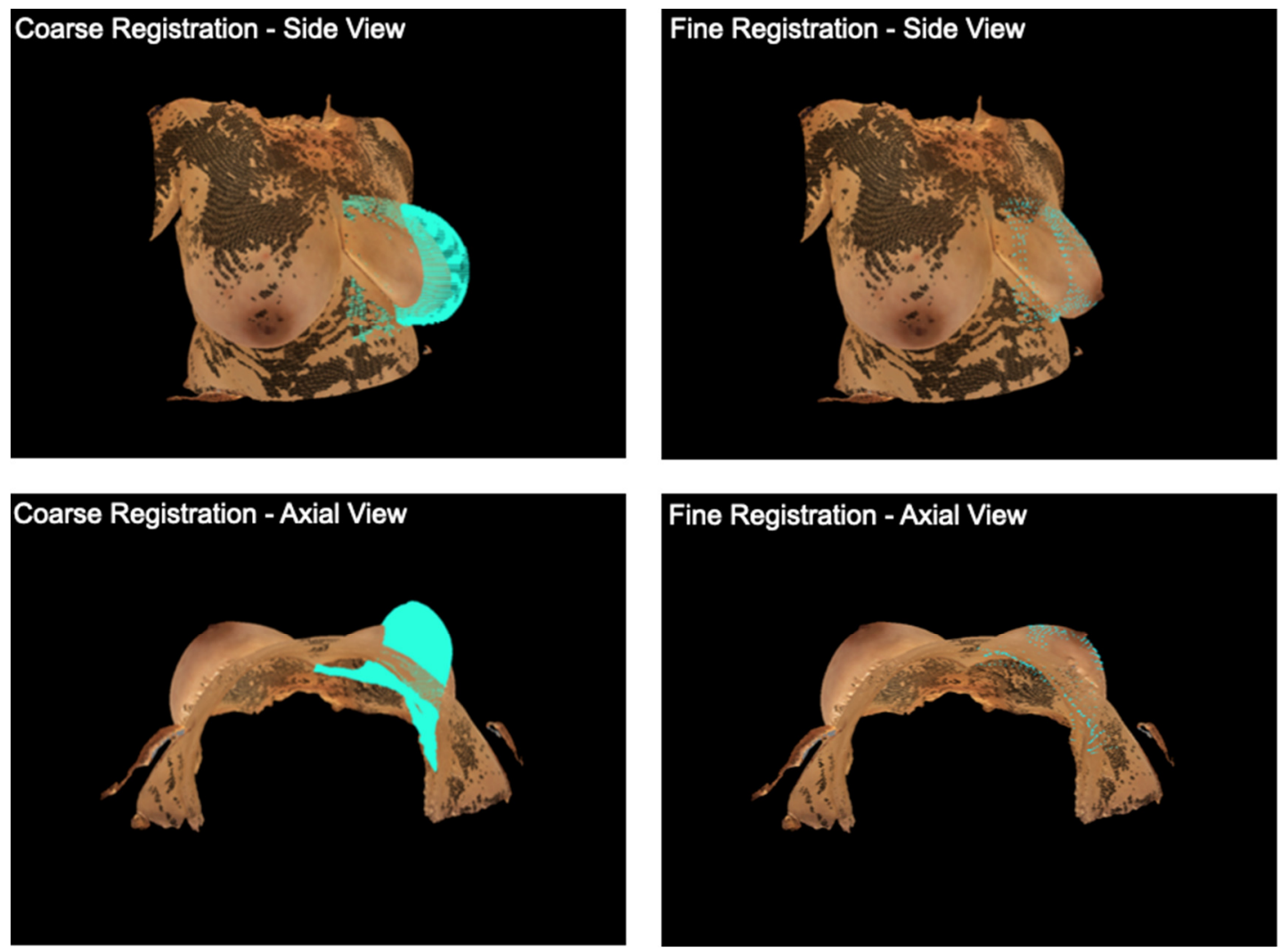

Fig. 4 - Results fo the coarse and fine registration steps of the fusion algorithm, using the automatic segmentations of the breast surface. 

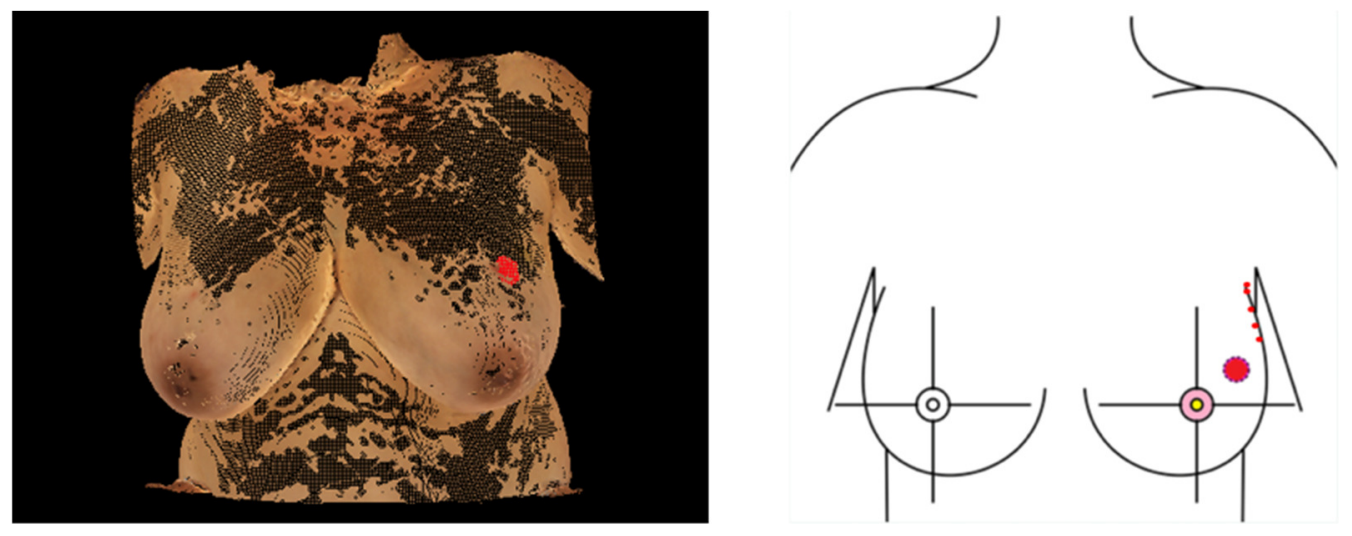

Fig.5 - 3D Scan with tumor (in red) after the multimodal registration with automatic segmentation of the MRI-T1w breast surface (on left), and schematic of the surgery annotation (on right).

\section{Results and Discussion}

Table 1 lists the results of the validation task of the 3D breast cancer models generated with proposed fusion algorithm using automatic breast surface segmentations. These personalized models had acceptable tumor locations for $75 \%$ of the patients. This means that if the surgeon relied on this preoperative location to plan the surgical intervention, the same surgical option and type of incision would have been selected. Moreover, for $50 \%$ of the patients, the projection of the tumor location on the 3D surface scan overlapped precisely with the carbon mark. The location of the tumor was also unaffected by using the automatic segmentations instead of the manual annotations: all 10 patients with GT annotations had acceptable locations and tumors registered to the correct quadrant, regardless of the breast surface (automatic or GT).

Table 1 - Validation of tumor locations.

\begin{tabular}{|c|c|c|c|}
\hline & Correct Quadrant & Acceptable Location & Overlap \\
\hline \# Patients & 20 & 18 & 12 \\
\hline \% Patients & $83.30 \%$ & $75 \%$ & $50 \%$ \\
\hline
\end{tabular}

These results are encouraging when exploring a new non-invasive tumor localization alternative, but despite being able to position the tumor on the correct quadrant for 20 out of 24 analyzed cases, 6 breast cancer models would still have unacceptable tumor locations. These include the 4 cases where the tumor was wrongly registered to a different quadrant or missed the expect quadrant intersection. A closer analysis of the results concluded that in all 6 models with unacceptable locations, the tumors were placed in a more medial location that the real location. This suggests that the fusion algorithm is unable to recover all de medial displacement of breast soft tissues caused by compression of the MRI.

In fact, the mechanics of this compression is still not fully understood. Some BM have tried to tackle this deformation in the past [11], but it remains an open problem.

Additionally, the distribution of correct quadrants for those 6 patients is: 3 tumors in the Upper Outer, 2 in the Lower Outer and 1 in the intersection of Upper Interior and Outer. The validation of the fusion agorithm [5] already suggested potential limitations of the fusion algorithm to deal with large lateral breast deformations caused by MRI coils, and showed slighlty larger TRE for breast surface markers limiting the outer quadrants. Some solutions for these limitations were also advanced: a short MRI breast acquisition in the supine position (after the normal MRI acquisition protocol in prone, the patient would change position to supine) would eliminate the medial displacement of tissues caused by the MRI coils and potentially facilitate the creation of 3D digital breast models both in supine or upright postion. But more important, the acquisition of a bigger sample of data with the breast surface markers following the placement of the validation protocol could boost the development of statistical and machine learning alternatives, leading to a better understanding of breast deformations and more accurate 3D modelling. 
Neverthless, a first feasibility test of 3D digital breast cancers used as a pre-operative location tool had already happened, which allowed direct tumor visualization inside the patient's breast using augmented reality on the operation theatre. The preliminary results are expected to be reported soon.

Finally, one can ask why merging the breast surface segmented on MRI-T1w if this structure is also segmented on the DCE-MRI sequence used to characterize the lesions. The rationale is two-fold: 1) multi-modal image registration of breast radiological images usually resorts to the 3D information of anatomical structures depicted on MRI-T1w. The tumor representation on a 3D digital breast model can be improved with the registration of additional exams such as mammography and ultrasound using available literature. 2) The performance of the fusion algorithm decreases drastically using breast surfaces that are shorter/partial on the coronal plane, which is the case of DCE-MRI segmentations. Take the results of the coarse registration step of the fusion algorithm using the original and new breast segmentations from MRI-T1w sequences (Fig.6) as example: original segmentations with less information on the coronal view (orange PCLs) are often wrongly rotated after the ICP algorithm. In these cases, the fusion algorithm lacks quasi-rigid portions of the torso that are shared by the 3D surface scan and the MRI and faces a more complex task: non-rigid registration with partial data.
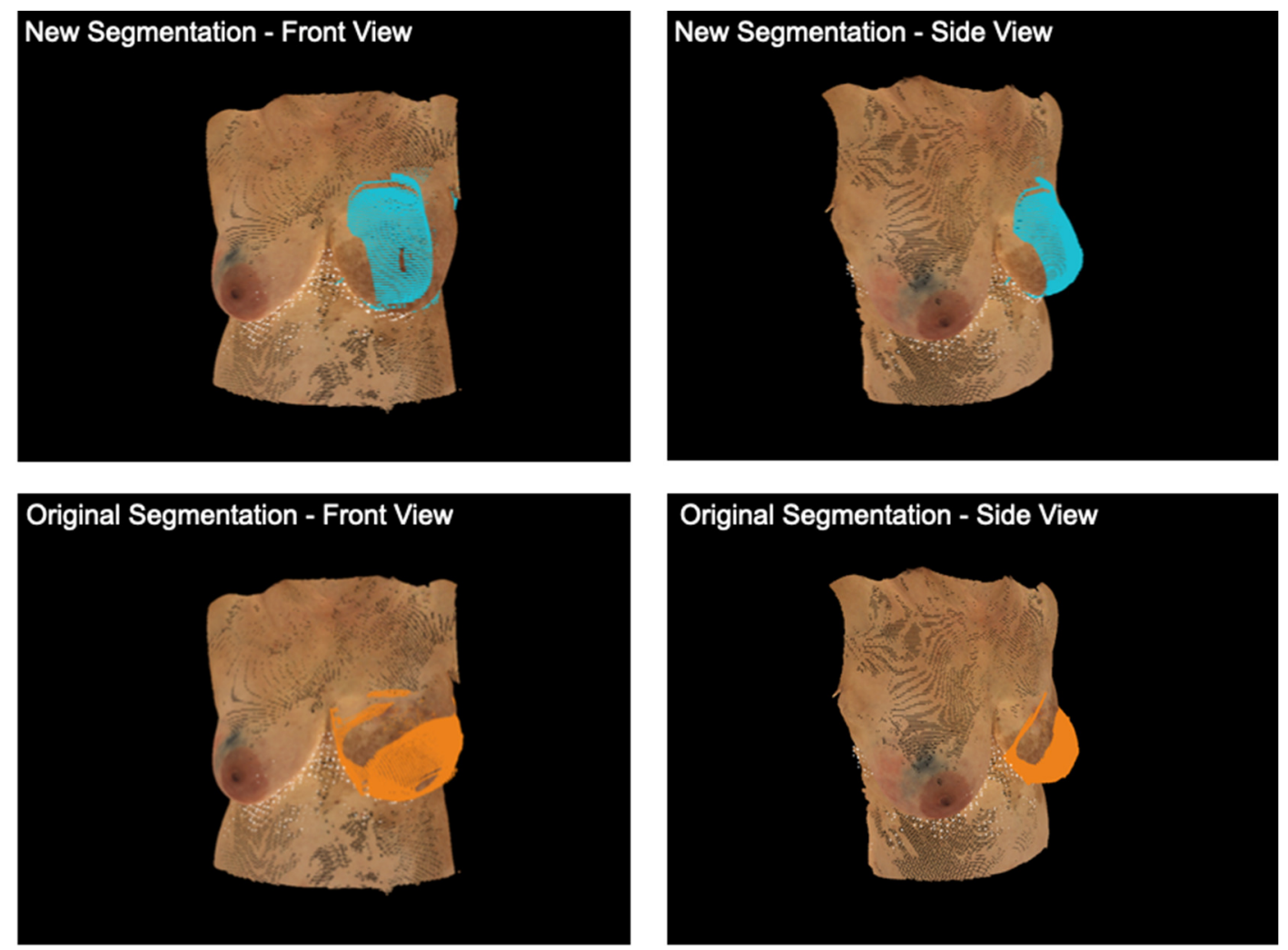

Fig. 6 - Coarse registration alignment results of the fusion algorithm using partial segmentations of the breast surface (original segmentations in orange) and the new segmentations that are closer to the GT (b/ue).

\section{Final Considerations}

Personalized 3D breast cancer models can be created using an automatic algorithm that segments breast surfaces on MRI-T1w sequences and performs the multimodal MRI/3D fusion in upright. These models provide acceptable pre-operative tumor locations for $75 \%$ of the patients and register the lesion to the correct quadrant in $83.3 \%$ of the cases. Therefore, they seem to be suitable for surgical planning tools that simulate expected surgery outcomes based on the tumor size and quadrant location such as [8] and [9]. However, the feasibility of those models as a non-invasive pre-operation localization tool might be hindered by the inability of the fusion algorithm to fully recover the medial displacement of breast tissues caused by MRI coils, but this requires further investigation. The proposed algorithm still uses the manual annotation of lesions on the DCE-MRI sequence, which is a simple and quick annotation task, but it can be automatized in the future. 


\section{Acknowledgments}

This work is financed by National Funds through the Portuguese funding agency, FCT - Fundação para a Ciência e a Tecnologia within project UIDB/50014/2020, and PhD grants number SFRH/BD/115616/2016 and SFRH/BD/135834/2018.

\section{References}

[1] F. Corsi, L. Sorrentino, D. Bossi, A. Sartani, and D. Foschi, "Preoperative Localization and Surgical Margins in Conservative Breast Surgery," International Journal of Surgical Oncology, vol. 2013, pp. 1-9, 2013

[2] R. C. Mayo, M. J. Kalambo, and J. R. Parikh, "Preoperative localization of breast lesions: Current techniques," Clinical Imaging, vol. 56, pp. 1-8, 2019.

[3] S. P. Oliveira, P. Morgado, P. F. Gouveia, J. F. Teixeira, S. Bessa, J. P. Monteiro, H. Zolfagharnasab, M. Reis, N. L. Silva, D. Veiga, M. J. Cardoso, H. P. Oliveira, and M. J. Ferreira, "Three-Dimensional Planning Tool for Breast Conserving Surgery: A Technological Review," Critical Reviews in Biomedical Engineering, vol. 46, no. 6, pp. 523-580, 2018.

[4] S. Bessa, P. H. Carvalho, and H. P. Oliveira, "Registration of Breast MRI and 3D Scan Data Based on Surface Matching," 2019 IEEE 16th International Symposium on Biomedical Imaging (ISBI 2019), 2019.

[5] S. Bessa, P. F. Gouveia, P. H. Carvalho, C. Rodrigues, N. L. Silva, F. Cardoso, J. S. Cardoso, H. P. Oliveira, and M. J. Cardoso, "3D digital breast cancer models with multimodal fusion algorithms," The Breast, vol. 49, pp. 281-290, 2020.

[6] M. Amano, T. Kitabatake, O. Nakata, Y. Ichikawa, R. Inaba, K. Ito, K. Ogura, Y. Ozaki, K. Kojima, S. Aoki, and R. Kuwatsuru, "Development of MRI Projection Mapping System for BreastConserving Surgery in the Operating Room: Preliminary Clinical Results in Invasive Breast Cancer," BioMed Research International, vol. 2020, pp. 1-9, 2020.

[7] J. F. Teixeira, S. Bessa, P. F. Gouveia, and H. P. Oliveira, "A Framework for Fusion of T1Weighted and Dynamic MRI Sequences," Lecture Notes in Computer Science Image Analysis and Recognition, pp. 157-169, 2020.

[8] T. Tökés, L. Torgyík, G. Szentmártoni, K. Somlai, A. Tóth, J. Kulka, and M. Dank, "Primary systemic therapy for breast cancer: Does the patients involvement in decision-making create a new future?," Patient Education and Counseling, vol. 98, no. 6, pp. 695-703, 2015. [DECISION MAKING]

[9] V. Vavourakis, B. Eiben, J. H. Hipwell, N. R. Williams, M. Keshtgar, and D. J. Hawkes, "Multiscale Mechano-Biological Finite Element Modelling of Oncoplastic Breast SurgeryNumerical Study towards Surgical Planning and Cosmetic Outcome Prediction," Plos One, vol. 11, no. 7, 2016.

[10] H. Zolfagharnasab, S. Bessa, S. Oliveira, P. Faria, J. Teixeira, J. Cardoso, and H. Oliveira, "A Regression Model for Predicting Shape Deformation after Breast Conserving Surgery," Sensors, vol. 18, no. 2, p. 167, 2018.

[11] J. H. Hipwell, V. Vavourakis, L. Han, T. Mertzanidou, B. Eiben, and D. J. Hawkes, "A review of biomechanically informed breast image registration," Physics in Medicine and Biology, vol. 61, no. 2, 2016. 